\title{
Feasibility Study of Using Engineered Cementitious Composite and High-Strength Bars in Rigid Bridge Piers Based on Seismic Vulnerability Analysis
}

\author{
Jie Li $\mathbb{D}$, Yuanhong Hu, Dayu Yang, Tengda Feng, Yan Liang $\mathbb{D}$, and Chenchen Tao \\ School of Civil Engineering, Zhengzhou University, Zhengzhou 450001, China \\ Correspondence should be addressed to Jie Li; lijie2007@zzu.edu.cn
}

Received 13 May 2020; Revised 14 August 2020; Accepted 8 September 2020; Published 27 September 2020

Academic Editor: Luigi Di Sarno

Copyright (c) 2020 Jie Li et al. This is an open access article distributed under the Creative Commons Attribution License, which permits unrestricted use, distribution, and reproduction in any medium, provided the original work is properly cited.

\begin{abstract}
The main function of pier is to transmit the load from superstructure to foundation reliably. Under earthquake action, the main failure reason of bridge is the damage of bridge pier. The application of some high-performance materials is helpful to improve the seismic performance of bridge piers. Based on seismic vulnerability analysis, this paper studies the feasibility of using engineered cementitious composite (ECC) and high-strength bars in bridge piers. Taking a rigid pier as an example, a nonlinear numerical model is established by OpenSees software. The reasonable replacement height of ECC in plastic hinge regions, stirrup ratio of pier section, and replacement rate of high-strength bars are obtained through the seismic performance analysis of the pier. Then, seismic vulnerability of rigid pier with ECC and high-strength bars is analyzed. The results show that it is feasible to improve the seismic performance of the piers by using ECC and high-strength bars. Considering the economic rationality, the replacement height of ECC in plastic hinge regions can be determined according to the curvature change point. For the rigid pier, the economical and reasonable volume stirrup ratio is $0.78 \%$. The ultimate curvature of RC/ECC pier bottom increases by $12.4 \%$ when the longitudinal bars of the pier are replaced by high-strength bars, and the energy dissipation capacity increases by $22.5 \%$ on average. Compared with the pier's original design, the exceedance probability of each limit state of the rigid pier with ECC and high-strength bars is significantly reduced. Its seismic performance is superior, and the risk of seismic damage is significantly reduced.
\end{abstract}

\section{Introduction}

Bridge is an important carrier for the normal operation of the transportation system and the development of the highway and railway transportation industry. The main function of pier structure is to transmit loads from superstructure to foundation reliably and effectively. In recent years, the investigation of earthquake damage shows that the main failure modes of piers under earthquake are bending failure, bending shear failure, and shear failure. Moreover, the fracture or damage of piers mostly occurs in the plastic hinge regions. The damage of piers further causes the whole girder to fall or collapse and other more serious disasters. According to Guidelines for Seismic Design of Highway Bridges [1], the ductility deformation capacity is increased by increasing the section size of piers and increasing the bars arrangement in the plastic hinge regions. However, these traditional ways often increase the difficulty of construction and the cost of engineering structure. Engineered Cementitious Composites (ECCs) are a kind of fiber-reinforced cement-based composite based on the principles of micromechanics and fracture mechanics [2]. When the fiber volume content is about $2 \%$, its ultimate tensile strain can reach more than $3 \%$, which has obvious strain hardening and multicrack characteristics, and the crack width is less than $0.1 \mathrm{~mm}$. ECC has good seismic performance and strong energy consumption capacity. It is a good seismic energy dissipating material mainly through large deformation, multicrack cracking, and strain hardening [3]. After decades of research by scholars at home and abroad [4-7], the ECC has been widely used in various engineering fields. In addition, the latest specifications for the Design of Highway Reinforced Concrete and Prestressed Concrete 
Bridges and Culverts (JTG 3362-2018) have eliminated the low-grade bars and supplemented the high-grade and largesize bars. So, the application of high-strength bars in bridge structures has become a major trend. It is necessary to discuss the replacement height of ECC in the pier, considering that most of the failure and fracture of pier components occur in the potential plastic hinge regions, and price of ECC materials is expensive. In addition, there are few research studies on the seismic vulnerability of piers using ECC and few research studies on the seismic performance of piers using highstrength bars and ECC at the same time. In this paper, a $36 \mathrm{~m}$ rigid frame bridge pier of a continuous rigid frame composite bridge is taken as an example. Under the longitudinal seismic excitation, the nonlinear finite element analysis model of the pier is established by OpenSees software. The potential height of plastic hinge is determined by the point of abrupt curvature in the curvature envelope of pier section along the pier body. The seismic performance of RC pier, RC/ECC composite pier, and full ECC pier is compared to study the feasibility of ECC application in piers. The influence of reasonable stirrup ratio and high-strength bars on the seismic performance of piers is analyzed. Finally, the seismic vulnerability of rigid frame piers with ECC and high-strength bars in the plastic hinge regions is analyzed and evaluated.

\section{Analysis of Replacement Height of ECC in Plastic Hinge Regions of Pier}

2.1. Example Pier and Design Parameters. In this paper, the example is a 6-span $(6 \times 60 \mathrm{~m}=360 \mathrm{~m})$ prestressed concrete rigid frame continuous beam bridge. The five piers are all separated rectangular solid piers. The section size of single pier is $2.2 \times 4.4 \mathrm{~m}$. The height of continuous pier is $9.17-16.12 \mathrm{~m}$, and $\mathrm{C} 40$ concrete is used. The two middle rigid frame piers are $36 \mathrm{~m}$ high and adopt C50 concrete. Their water cement ratio is 0.5. HRB335 (hot rolled ribbed bars with yield strength of $335 \mathrm{MPa}$ ) bars with a diameter of $\varphi 32$ are used as the longitudinal bars. HRB335 bars with a diameter of $\varphi 16$ are used as stirrup. The longitudinal bar ratio is $1.12 \%$, and the volume stirrup ratio is $0.54 \%$. The thickness of the concrete cover is $90 \mathrm{~mm}$. The foundation form is open cut and rigid expansion foundation. The site condition is class II, with a seismic fortification intensity of $7^{\circ}$ (according to the Chinese standard).

\subsection{Material Constitutive and Numerical Analysis Model.} The nonlinear analysis model of the pier is established by OpenSees. In the material library of the software, Steel02 material is used to simulate the bar material. Concrete 01 material is used to simulate ordinary concrete material. The ECC material is simulated by concrete 02 material that can consider the tension section of concrete material.

\subsubsection{Material Constitutive Model}

(1) Steel Bar Constitutive Model. In OpenSees, steel02 material is based on the Giuffre-Menegotto-Pinto model to simulate the working mechanism of steel bars. The calculation formula of the model is relatively simple, and it is in good agreement with the test results of steel bars materials, so it has wide applicability. In the paper, the bars are based on the mentioned model, and their constitutive models are shown in formula (1). The material constitutive parameter in the OpenSees model is shown in Table 1:

$$
\sigma=R_{s} \varepsilon+\frac{\left(1-R_{s}\right) \varepsilon}{\left(1+\varepsilon^{R_{b}}\right)^{1 / R_{b}}} .
$$

In the formula, $R_{S}$ is the strain-hardening coefficient and $R_{b}$ is the parameter considering Bauschinger effect, which indicates the strain-softening degree of steel bars.

The strain-hardening coefficient $b$ in Table 1 is the parameter $R_{S}$ in formula (1). The format of steel02 material is "uniaxialmaterial steel02 \$mattag \$matTag \$fy \$E \$b \$R0 $\$ c R 1$ \$cR2" in the OpenSees. The isotropic hardening of steel bars is considered by different values of $R 0, R 1$, and $R 2$. In this paper, the parameters of steel02 are as follows in the OpenSees: uniaxialmaterial steel02 \$mattag 33500 2000000000.01180 .9250 .15 [8].

(2) Constitutive Model of Ordinary Concrete and ECC. The Mander constitutive model is a constitutive relation curve including the rising and falling stages. Considering the influence factors comprehensively, the Mander constitutive model is suitable for different cross-section forms, such as circular, square, and wall type. Therefore, the Mander model is more suitable for nonlinear simulation of structural members. In the OpenSees material library, there are two kinds of concrete materials based on the Mander constitutive model: concrete 01 material and concrete 02 material. Concrete 02 material considers the tension stage of concrete material. Therefore, concrete 01 material is used to simulate ordinary concrete material, and concrete 02 material is used to simulate ECC material. In this paper, the parameters of concrete 01 and concrete 02 are as follows in the OpenSees: uniaxialMaterial Concrete01 \$matTag \$fpc \$epsc0 \$fpcu \$epsu; uniaxialMaterial Concrete02 \$matTag \$fpc \$epsc0 \$fpcu \$epsu \$lambda \$ft \$ets.

Among the above parameters, $\$$ fpc and $\$$ epsc0 are the peak of the stress and strain, and \$fpcu and \$epsu are the yield stress and strain. \$lambda is the hysteretic characteristic parameter, and the value is the ratio of the slope of the unloading curve to the slope of initial stretching curve, which is generally 0.1 by default. $\$ \mathrm{ft}$ is the ultimate tensile strength. \$ets is the tensile-softening stiffness. See formula (2) for the uniaxial tension constitutive relation of ECC material [9]:

$$
\sigma_{t}= \begin{cases}E \varepsilon_{t}, & \left(\varepsilon_{t} \leq \varepsilon_{t 0}\right), \\ \sigma_{t 0}+\left(f_{t 1}-\sigma_{t 0}\right) \frac{\varepsilon_{t}-\varepsilon_{t 0}}{\varepsilon_{t 1}-\varepsilon_{t 0}}, & \left(\varepsilon_{t 0}<\varepsilon_{t} \leq \varepsilon_{t 1}\right), \\ f_{t 1}\left(\frac{\varepsilon_{t}-\varepsilon_{m}}{\varepsilon_{t 1}-\varepsilon_{t u}}\right), & \left(\varepsilon_{t 1}<\varepsilon_{t} \leq \varepsilon_{t u}\right), \\ 0, & \left(\varepsilon_{t}>\varepsilon_{t u}\right) .\end{cases}
$$


TABLE 1: Material parameter value of steel bars.

\begin{tabular}{lccc}
\hline Parameter & Modulus of elasticity $E_{S}(\mathrm{MPa})$ & Yield strength $f_{y}(\mathrm{MPa})$ & Strain-hardening coefficient $b$ \\
\hline HRB335 & 200000 & 335 & 0.01 \\
\hline
\end{tabular}

In the formula, $E$ is the modulus of elasticity, $\sigma_{t 0}$ and $f_{t 1}$ are cracking stress and tensile strength, respectively, $\varepsilon_{t 0}$ and $\varepsilon_{t 1}$ are the corresponding strain values, and $\varepsilon_{t u}$ is the ultimate tensile strain. The values are $34500 \mathrm{MPa}, 3 \mathrm{MPa}$, and $3.5 \mathrm{MPa}, 0.02 \%, 1.77 \%$ and $2 \%$, respectively.

In consideration of the residual strength, the ECC constitutive relation under uniaxial compression adopts formula (3) [9]:

$$
\sigma= \begin{cases}f_{c 0}\left[2\left(\frac{\varepsilon}{\varepsilon_{c 0}}\right)-\left(\frac{\varepsilon}{\varepsilon_{c 0}}\right)^{2}\right], & \left(0 \leq \varepsilon \leq \varepsilon_{c 0}\right), \\ \frac{f_{c 0}}{\varepsilon_{c}-\varepsilon_{c 0}}\left(\varepsilon_{c}-0.2 \varepsilon_{c 0}-0.8 \varepsilon\right), & \left(\varepsilon_{c 0}<\varepsilon \leq \varepsilon_{c}\right), \\ 0.2 f_{c 0}, & \left(\varepsilon>\varepsilon_{c}\right) .\end{cases}
$$

In the formula, $f_{c 0}$ is the peak compressive stress, $\varepsilon_{c 0}$ is the peak compressive strain, and $\varepsilon_{c}$ is the strain at which the stress decreases to $20 \%$ of the peak stress. The values are $32.4 \mathrm{MPa}, 0.005$, and 0.01 , respectively.

2.2.2. Analysis Model. The finite element model of pier is one node per meter along the direction of pier height, with 37 nodes in total. The lumped mass method is used to calculate the structural mass matrix. The weight of the superstructure of the bridge is converted into the mass of the pier top node, and the mass of the rest nodes is the mass of the pier body. The rigid enlarged foundation is simulated by consolidating connection between pier bottom and foundation. Considering the consolidation of pier and beam of continuous rigid frame bridge, the boundary constraint diagram of main beam to pier top is shown in Figure 1(a). Through the forced displacement analysis, the $F$ (Figure 1(b)) and $M$ (Figure 1(c)) of the main beam to the pier top in the plane along the bridge direction are obtained. The zero-length element is used to simulate the constraint of the main beam to the pier top.

The pier adopts the beam column element based on the flexibility method proposed by Neuenhofer [10]. The results show that the ideal accuracy can be achieved when the number of integration points is set to 3-4 based on the flexibility method [11]. Therefore, three integration points are selected. In addition, the flexibility method has little effect on the accuracy of the calculation, so the number of fibers in the section of pier is more than 40 , which can meet the accuracy requirements enough. In order to facilitate the replacement of steel and concrete materials, the fiber section is divided into two parts $[12,13]$.

In the OpenSees, the compressive strength of concrete cylinder needs to be input, and the conversion coefficient between the compressive strength of concrete cylinder and that of concrete cube is 0.85 . Considering the restraint effect of stirrup on concrete, the concrete outside and inside stirrup are modeled, respectively; that is, the concrete is simulated as confined concrete and unconstrained concrete, respectively. Based on the Mander model, the calculation of the constitutive relation of core concrete is as follows:

$$
\begin{aligned}
f_{c c}^{\prime} & =K f_{c}^{\prime}, \\
\varepsilon & =\left[5\left(\frac{f_{c c}^{\prime}}{f_{c}^{\prime}}-1\right)+1\right] \bullet \varepsilon_{c o}, \\
\varepsilon_{c u} & =0.004+1.4 \frac{\rho_{s} f_{y} \varepsilon_{s u}}{f_{c c}^{\prime}}, \\
K & =1+\frac{\rho_{s} \cdot f_{v h}}{f_{c}^{\prime}} .
\end{aligned}
$$

In the above formulas, $f_{c c}^{\prime}$ and $\varepsilon_{c u}$ are peak stress and peak strain of confined concrete, respectively, $f_{c}^{\prime}$ is the compressive strength of unconstrained concrete cylinder, $K$ is the effective constraint coefficient, which is $1.1, \rho_{S}$ is the volume stirrup ratio, $f_{y}$ is the maximum tensile stress of stirrup, $f_{v h}$ is the yield strength of stirrup, and $\varepsilon_{s u}$ is the strain corresponding to the maximum tensile stress of stirrup, generally taken as 0.09 .

2.3. Height of Plastic Hinge Regions of Rigid Pier. In order to ensure that the pier components have enough bending and ductile deformation capacity under the dynamic action, the code requires that the pier section size should be increased appropriately in the seismic design, or the stirrup should be densified in the plastic hinge regions. However, these measures will increase the difficulty of construction, and the effect of ductility improvement is limited. ECC material can make structural members have strong seismic resistance, but ECC is about 10 times the cost of ordinary concrete material with the same volume. Considering the performance and economic reasons, the replacement height of plastic hinge regions should be determined first. On this basis, the reasonable height of replacement ECC for piers is discussed. Considering the relationship between the curvature and displacement of the pier column, the displacement of the middle and low pier column can be obtained by the integral of curvature function along the direction of the pier body $[12,14,15]$. However, the curvature function of the actual pier shaft is difficult to determine, and the calculation involved is also very complex. Based on Huang's research [16], 15 natural seismic waves are downloaded from the Pacific Earthquake Engineering Research Center (PEER) database according to the conditions such as the average shear wave velocity $V_{s 30}$ and response spectrum characteristics of class II site. Under the longitudinal seismic excitation, the OpenSees finite element software is used for 


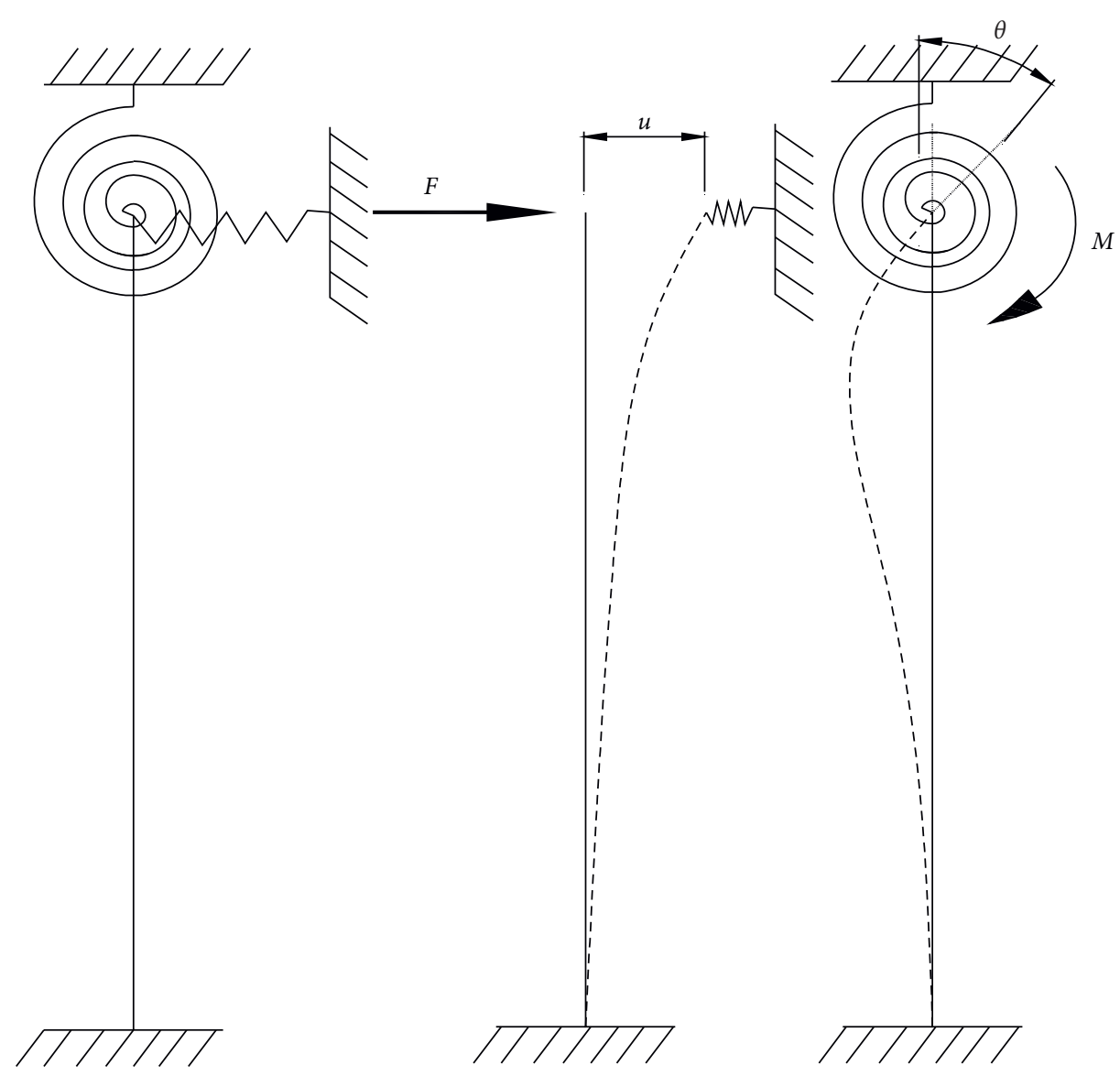

(a)

(b)

(c)

FIGURE 1: Boundary constraint calculation of the rigid pier top.

nonlinear analysis. The curvature envelope of rigid frame pier section along the pier body is obtained, as shown in Figure 2.

The purpose of this study is mainly to discuss the feasibility of using ECC for piers. Due to limited space and research purposes, the length of plastic hinge and potential plastic hinge regions are only based on numerical analysis. The location of curvature mutation point is selected as the region and length of plastic hinge. It can be seen from Figure 3 that under the longitudinal seismic excitation, the maximum curvature along each section of the pier appears at the top and bottom of the pier; that is, the plastic hinge regions may be at the top and bottom of the pier. The length of the plastic hinge can be obtained by taking the mutation points of curvature as the criterion of judging the region of the plastic hinge. In Figure 3, the coordinates of mutation points are $(-0.00303,35.0957),(0.002839$, $35.0609),(-0.0029564,0.93913)$, and (0.00297998, 0.921739). In the OpenSees model, the length of the element is $1 \mathrm{~m}$, and the mutation points of the pier section along the curvature envelope of the pier appear in element 1 and element 36. In order to facilitate the calculation, $1 \mathrm{~m}$ is selected as the height of the plastic hinge regions at the top and bottom of the pier.

2.4. Analysis of the Replacement Height of ECC. Based on the above analysis, it is considered that the potential plastic hinge regions are only at the top and bottom of the pier. In order to determine the economic and reasonable replacement height of ECC for piers, OpenSees is used to establish four cases of the replacement height of ECC for the top and bottom of pier, which are $0 \mathrm{~m}, 0.5 \mathrm{~m}, 1 \mathrm{~m}$, and full height, respectively. The following four types of pier are, respectively, referred to as RC pier, $0.5 \mathrm{~m} \mathrm{RC/ECC} \mathrm{pier,} 1 \mathrm{~m} \mathrm{RC/}$ ECC pier, and full ECC pier. Three groups of small and medium earthquake waves in the strong earthquake database of PEER are selected for longitudinal seismic excitation, and the nonlinear time-history analysis of the pier model is carried out to obtain the seismic response of pier top and pier bottom sections. Due to limited space, Figure 3 only shows the displacement time-history curve and momentcurvature hysteretic curve of RC, $1 \mathrm{~m} \mathrm{RC/ECC,} \mathrm{and} \mathrm{full} \mathrm{ECC}$ pier top under the action of one seismic wave.

It can be seen from Figure 3 that the seismic response of RC pier is larger than that of RC/ECC pier or ECC pier, but the difference between the seismic response of ECC pier and RC/ECC pier is small. The results show that if the pier is replaced by ECC, the displacement of pier top will be slightly reduced, and the maximum curvature will be significantly reduced. In order to explore the rule of each pier under strong earthquake excitation, the strong earthquake records are obtained by adjusting the natural wave amplitude to 1.8 times, and 4 kinds of pier models are input longitudinally. Due to limited space, under the 


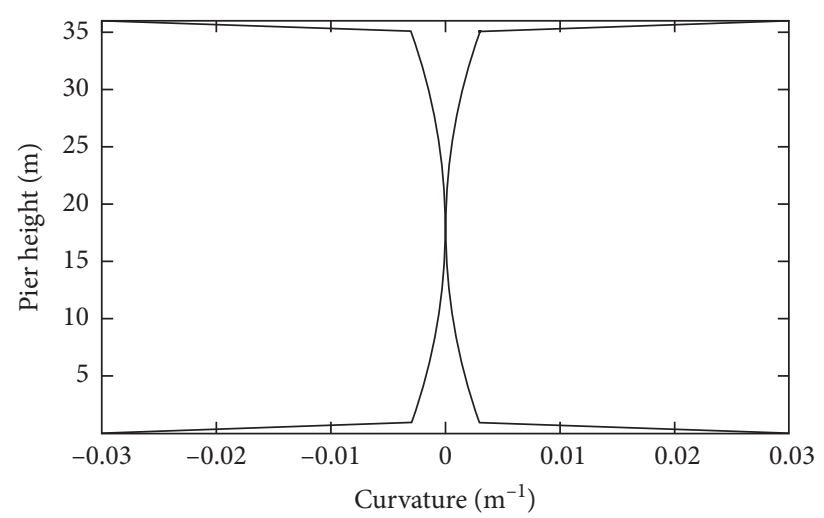

Figure 2: Curvature envelope of rigid frame pier section along pier.

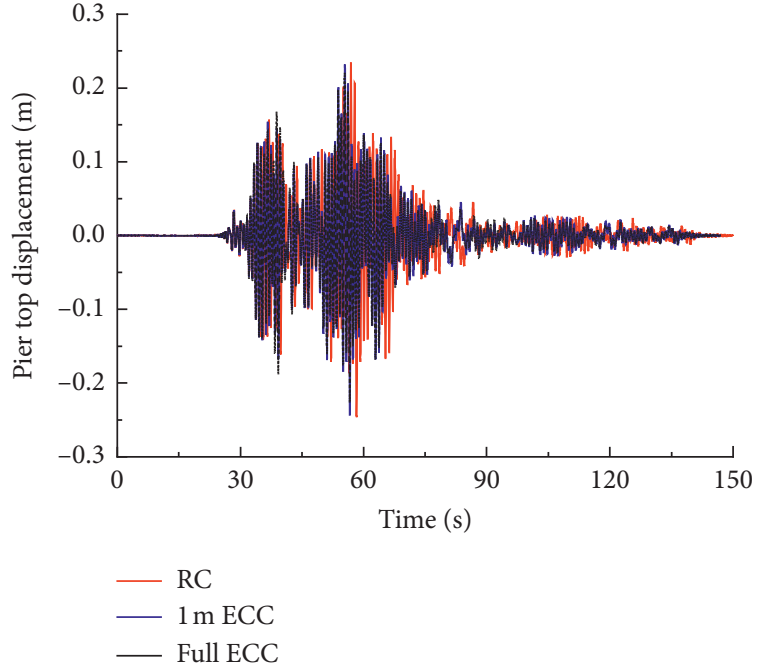

(a)

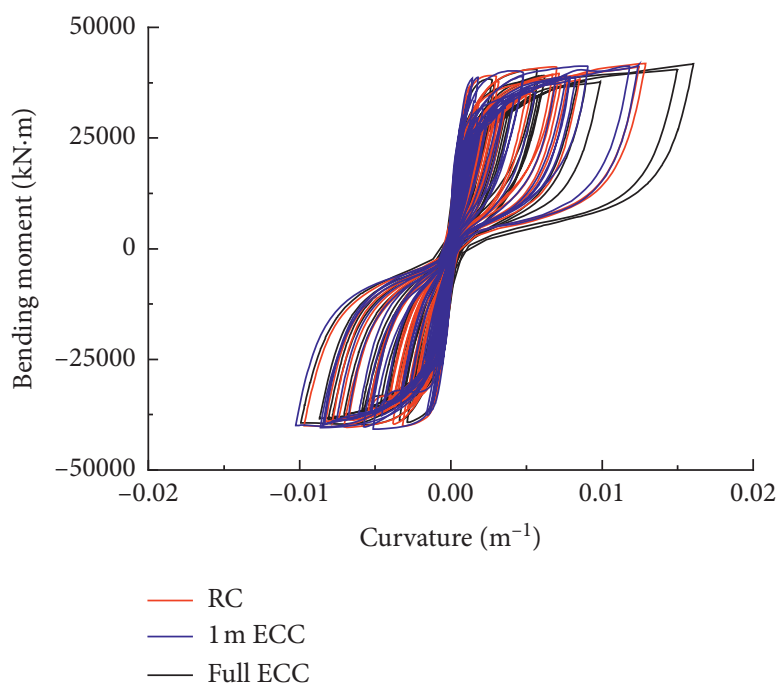

(b)

FIGURE 3: Seismic response of the piers: (a) time-history curve of pier top displacement and (b) moment-curvature hysteretic curve.

same strong earthquake wave, Figure 4 shows the displacement time-history curve and moment-curvature hysteretic curve of RC, $0.5 \mathrm{~m} \mathrm{RC/ECC,} 1 \mathrm{~m} \mathrm{RC/ECC}$, and full ECC pier top.

It can be seen from Figure 4(a) that under the action of amplitude modulated seismic wave, the largest pier top displacement in four cases is $0.283707,0.300668,0.311571$, and 0.317915 . The pier top displacement of the full ECC is the largest. It can be seen from Figure 4(b) that there is no significant difference in the maximum bending moment of the pier, but there is a significant difference in the maximum curvature. Among them, the maximum curvature of full ECC piers is the largest, and the maximum curvatures of ECC piers with plastic hinge region replacement height of $0.5 \mathrm{~m}$ and $1 \mathrm{~m}$ are significantly different. However, the maximum curvatures of the pier with plastic hinge region replacing ECC and pier full height replacing ECC are similar. The analysis also shows that (1) RC/ECC piers have fuller hysteresis loops, and the area of hysteresis loops is significantly higher than that of RC piers, (2) when ECC materials are used in the plastic hinge regions, the ductility coefficient of pier components can be greatly improved, and the plastic deformation ability is better, and (3) when ECC material is used in the plastic hinge regions, the equivalent viscous damping coefficient of the pier increases, and the energy dissipation capacity of the pier is better.

Therefore, ECC can significantly reduce the seismic response of piers under the action of medium and small earthquakes. Under the action of strong earthquake, the maximum displacement of pier top increases slightly. The maximum curvature of pier bottom increases obviously, but the maximum moment of pier bottom does not show obvious change. It is consistent with Jia et al.'s [17] research conclusion. Whether it is a small earthquake or a strong earthquake, the maximum curvature of the pier with $0.5 \mathrm{~m}$ and $1 \mathrm{~m}$ replaced by ECC material in the height of plastic hinge regions is obviously different. For the two kinds of piers, replaced by ECC at the plastic hinge regions and the whole height, the difference of maximum curvature is very small. Considering the economy, only replacing ECC within the height of plastic hinge regions (in this paper, replacing 


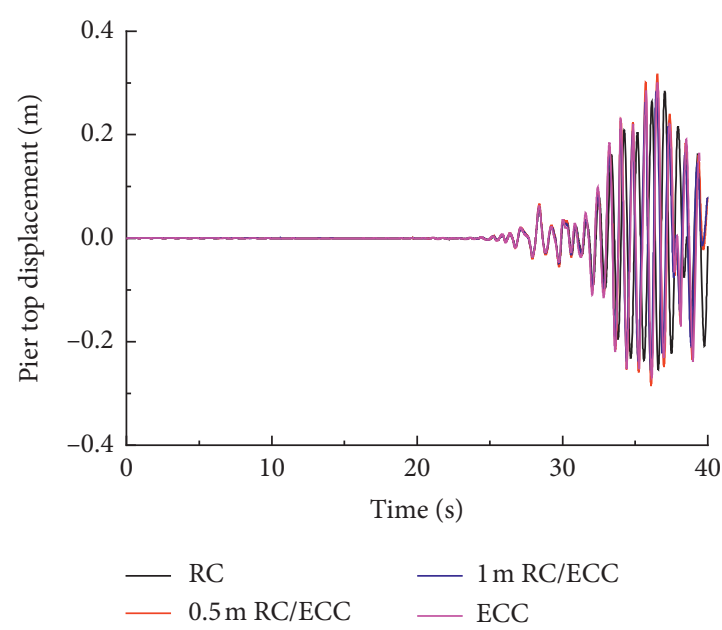

(a)

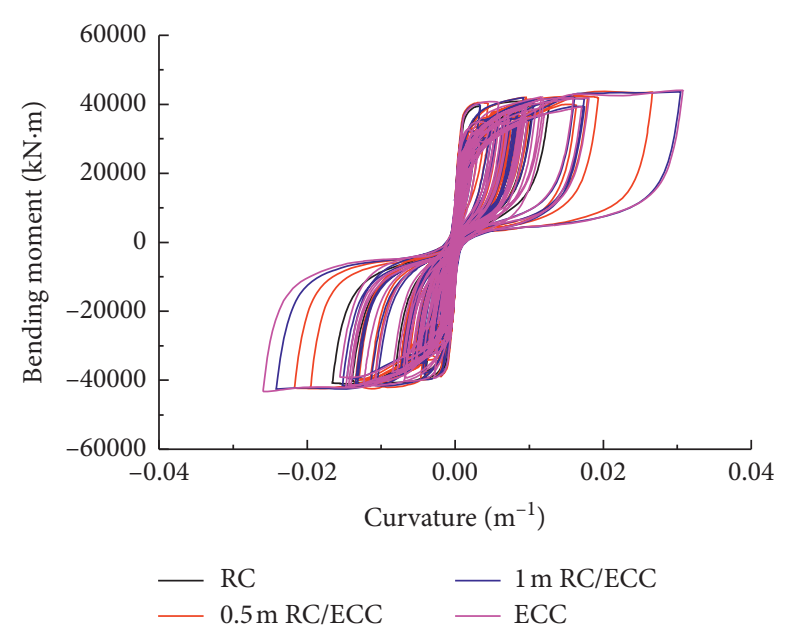

(b)

FIGURE 4: Seismic response of the piers: (a) time-history curve of pier top displacement and (b) moment-curvature hysteretic curve.

$1 \mathrm{~m}$ concrete for pier top and pier bottom, respectively) can significantly improve the seismic capacity of pier.

\section{Seismic Analysis of RC/ECC Pier with High- Strength Bars}

3.1. Reasonable Stirrup Ratio of ECC Pier. According to Detailed Rules for Seismic Design of Highway Bridges [1], in the area where the seismic fortification intensity is $7^{\circ}$ or above, the potential plastic hinge regions of the bridge pier column need to be densified with stirrups. The height of the densified area shall not be less than 1 times of the section width of the bending direction of the bridge pier column, and the stirrup ratio in the height of the densified area shall be at least $0.4 \%$. Because of the analysis of longitudinal seismic input in this paper, the section width in the bending direction of the pier is $2.2 \mathrm{~m}$, and the potential plastic hinge regions are $1 \mathrm{~m}$, so $h=2.2 \mathrm{~m}$ is taken as the stirrup densification height. The stirrup ratio of the example pier is $0.54 \%$, and the minimum stirrup ratio of the densified area is not less than $0.4 \%$. In order to determine the reasonable stirrup ratio in the densified area, the stirrup ratio of $0.4 \%, 0.54 \%$, $0.78 \%$, and $1.02 \%$ are selected for comparative analysis by changing the stirrup spacing in the densified area. By comparing the seismic response under the action of small and medium earthquakes, the reasonable stirrup ratio of $\mathrm{RC} /$ ECC composite piers in the densified area after the plastic hinge regions is replaced by ECC material is determined qualitatively. Due to limited space, Figure 5 only gives a comparison of hysteretic curves under the action of a real seismic wave.

It can be seen from Figure 5 that the maximum curvature demand response of RC/ECC composite rigid frame pier under the action of this seismic wave is $0.0147203,0.0133501,0.0123374$, and 0.0121078 after the concrete in plastic hinge regions is replaced with ECC. Under the action of ground motion, the stirrup ratio in the height of dense area has no obvious influence on the bending capacity of the example pier. However, due to the increase of stirrup ratio, the ductility of pier becomes stronger, and the maximum curvature demand of pier will decrease with the increase of stirrup ratio under the same earthquake. When the stirrup ratio is $0.78 \%$ and $1.02 \%$, the difference of the maximum curvature demand is small; that is to say, when the stirrup ratio is $0.78 \%$, the ductility of the bridge pier is significantly enhanced. In consideration of the economy and improvement effect, $0.78 \%$ is selected as the stirrup ratio of the densified zone in the subsequent analysis.

3.2. Analysis of Replacement Rate of High-Strength Bars. In view of the excellent mechanical properties of highstrength bars, taking as an example the pier with $0.78 \%$ stirrup ratio in the densified area and the concrete in the plastic hinge regions replaced by ECC, the influence of replacement ratio of high-strength bars in the section of pier on the seismic performance is further studied. The original design of the pier has 82 longitudinal bars in total. The longitudinal bars in the model are replaced by HRB500 highstrength bars. The replacement rate of longitudinal bars is $25 \%, 50 \%$, and $100 \%(20,40$, and all longitudinal bars are replaced). Then, the nonlinear time-history analysis of the bridge pier is carried out by inputting seismic wave. Figure 6 only gives the moment-curvature hysteretic curve calculated by one of the seismic waves.

It can be seen from Figure 6 that the maximum curvature values of RC/ECC pier with different highstrength bars replacement rates $(0 \%, 25 \%, 50 \%$, and $100 \%)$ are $0.0304698,0.0333292,0.0341029$, and 0.0355749 , and the maximum bending capacity is 43687.1, 45880.6, 47835.7 , and $51778.6 \mathrm{kN} \mathrm{m}$, respectively. The results of other seismic wave calculation are similar. That is to say, under the action of amplitude modulated earthquake, the maximum curvature and ultimate bending capacity of RC/ ECC pier increase with the increase of replacement rate of 


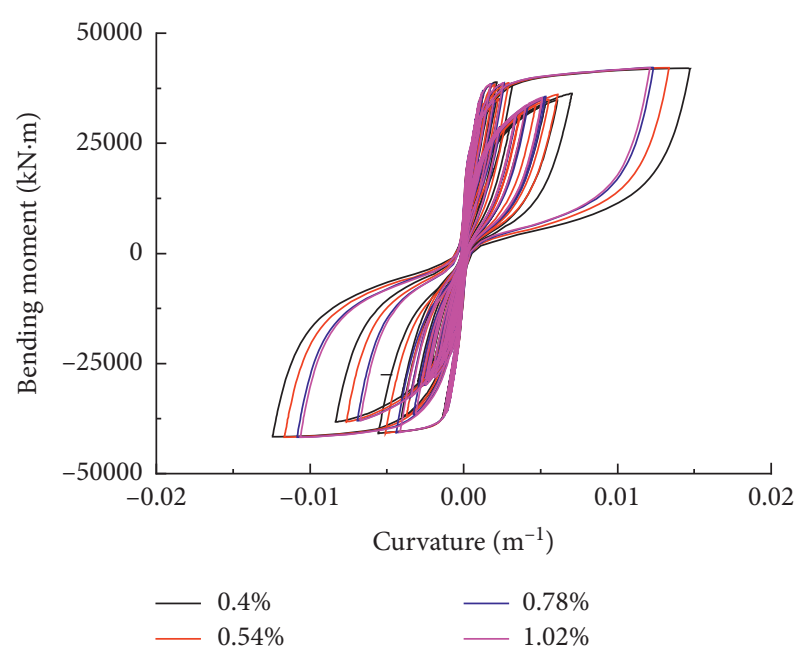

Figure 5: Comparison of hysteretic curves with different stirrup ratios.

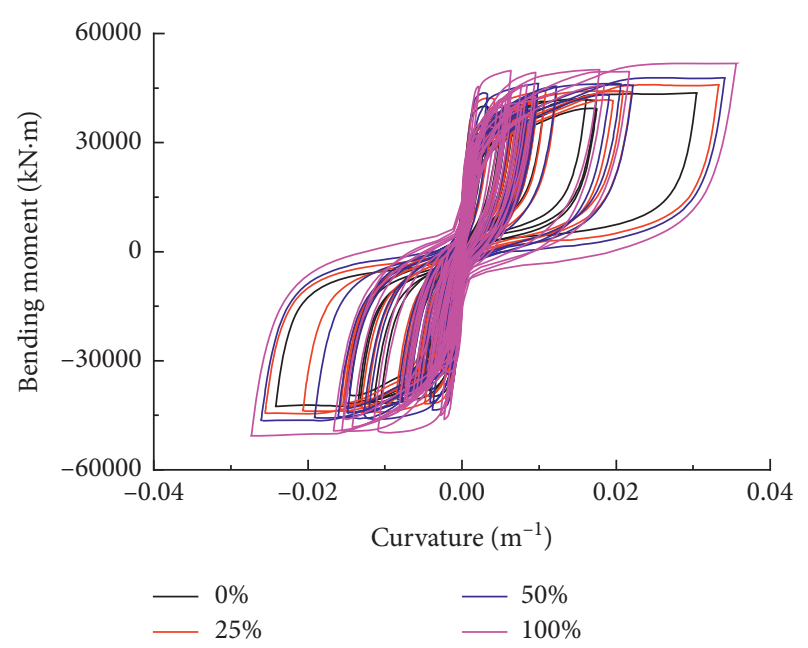

Figure 6: Hysteretic curve of RC/ECC pier with different replacement rates of high-strength bars.

high-strength bars. In addition, the replacement rate of high-strength bars also has a significant impact on the residual deformation of the structure. It is mainly because the high-strength bars have the characteristics of high strength and good ductility and have the similar elastic modulus with the ordinary bars. Therefore, RC/ECC pier with high-strength bars have better ductility, and the bending capacity increases with the replacement of highstrength bars.

\section{Seismic Vulnerability Analysis of RC/ECC Pier with High-Strength Bars}

HRB500 high-strength bars are used as the main bars of the whole section of the pier, the stirrup ratio in the dense area is $0.78 \%$, and the concrete in the plastic hinge regions with the height of $1 \mathrm{~m}$ at the top and bottom of the pier is replaced by ECC. The following is a comparative analysis of seismic vulnerability of the pier.

\subsection{Determination of Earthquake Action Input and Damage Index}

4.1.1. Action Input of Earthquake. According to the capacity-demand ratio proposed by Pan et al. [18], 160 seismic waves are selected for seismic vulnerability analysis. In addition, the peak ground acceleration (PGA) of the selected seismic wave is consistent with the peak value of frequent and rare earthquakes at the pier location. According to the code, the average shear wave velocity $V_{s 30}$ corresponding to the class II site of the bridge site is used to select the wave, so as to ensure that the response spectrum of the selected seismic wave is consistent with the spectrum characteristics of the class II site as much as possible. In order to make the pier bear enough earthquake energy, the seismic wave duration is taken as $5-10$ times of the basic period of the structure. Since PGA is only related to the ground motion itself and has nothing to do with the characteristics of the structure, it can be selected as the earthquake ground motion intensity index for seismic vulnerability research.

4.1.2. Damage Index. HAZUS 99 [19], a user manual issued by the Federal Emergency Management Agency of the United States, proposes a method for seismic vulnerability assessment. The performance objectives of the bridge under the action of earthquake are divided into five damage levels: basically intact, slightly damaged, moderately damaged, severely damaged, and completely collapsed, as shown in Table 2. These four limit states correspond to $L S_{i}(i=1,2,3$, and 4$)$ : minor damage $L S_{1}$, moderate damage $L S_{2}$, severe damage $L S_{3}$, and complete failure $L S_{4}$. The HAZUS 99 classification method is careful and reasonable. The following method is used to define the limit state of piers under earthquake. It should be noted that for the purpose of this study, the top and bottom bending moment-curvatures of rigid pier are used to characterize the limit state without considering the uncertainty of the limit state.

The location of plastic hinge of rigid frame pier appears at the top and bottom of pier. Therefore, the top and bottom sections of the RC pier and high-strength reinforced RC/ ECC pier (hereinafter referred to as RC/ECC pier) are all control sections, and the bending moment-curvature analysis is carried out, respectively. According to Niu's paper [20], with the increase of service time, the flexural capacity of the section will decrease. The equivalent yield moment $\left(M_{y}\right)$ is also significantly reduced, but the change of the equivalent yield curvature $\left(\phi_{y}\right)$ of the section is relatively small, so the change can be ignored. With the help of XTRACT software, the moment-curvature analysis of the pier control section is carried out, and the $M-\phi$ curve of the pier is obtained. At the same time, the curvatures corresponding to four damage states (minor damage, moderate damage, severe damage, and complete failure) of $\mathrm{RC}$ pier and RC/ECC pier are obtained, which are taken as the basis for vulnerability analysis and evaluation, as shown in Table 3. 
Table 2: Damage grade of pier.

\begin{tabular}{lcc}
\hline Destruction level & Destruction description & Quantitative description of damage indicators \\
\hline Basically intact & Small cracks in concrete & $\phi_{y}<\phi_{y}^{\prime}$ \\
Minor damage & First yield of longitudinal bar & $\phi_{y}^{\prime} \leq \phi<\phi_{y}$ \\
Moderate damage & Longitudinal bar reaches theoretical yield value & $\phi_{y} \leq \phi<\phi_{\mathrm{d}}$ \\
Severe damage & Core area concrete reaches the maximum bearing capacity & $\phi_{d} \leq \phi<\phi_{m}$ \\
Complete failure & Core area concrete crushed & $\phi \geq \phi_{m}$ \\
\hline
\end{tabular}

\subsection{Comparative Analysis of Seismic Vulnerability of Pier}

4.2.1. Capacity-Demand Ratio Analysis. Capacity-demand ratio analysis model is the first reliability theory analysis method proposed by Pan et al. [18]. In this model, the ratio of the earthquake demand in the time-history analysis results to the corresponding damage limit index of each damage state is taken. At the same time, the ratio and seismic intensity are plotted in the same logarithmic coordinate system, and the regression analysis of the discrete data points is carried out by using the quadratic polynomial function. The regression formulas of capacity-demand ratio in this paper are as follows (8)-(11):

$$
\begin{gathered}
\text { Minor damage } y=0.0148 x^{2}+1.2685 x+0.5515 \\
\text { Moderate damage } y=0.0148 x^{2}+1.2685 x+0.5515 \\
\text { Severe damage } y=0.0148 x^{2}+1.2685 x-0.9999 \\
\text { Complete damage } y=0.0148 x^{2}+1.2685 x-1.0743
\end{gathered}
$$

where $y=\ln (D / C), D / C$ is the ratio of curvature demand $\phi$ to the curvature damage index under specific failure state, and $x=\ln$ (PGA). According to the related research, the capacity-demand ratio obtained by this method has lower discreteness [18]. The fitting accuracy is higher, and the result is more objective. In view of this, the mean value $\lambda$ of the capacity-demand ratio is expressed as the quadratic fitting function of $\ln$ (PGA). The standard deviation $\sigma$ of the regression curve fitting is the standard deviation of the capacity-demand ratio of the control section at the bottom of the pier. At the same time, the seismic strength grade used in time-history analysis is limited. Carrying out the internal difference for the seismic strength grade, according to the internal difference strength parameters, the corresponding mean value and standard deviation are calculated. Table 4 shows the mean value and standard deviation after fitting under the condition of slight damage of pier bottom section.

4.2.2. Comparison of Seismic Vulnerability of Pier. Because $\ln (D / C)$ obeys normal distribution, $\lambda$ and $\sigma$ values can be obtained by putting different PGA into the regression formula. Then, the exceedance probability $P_{f}$ is obtained. Equations (12)-(15) give the formula for calculating the exceedance probability $P_{f}$ of piers:

$$
P_{f}\left(L S_{1}\right)=\Phi\left[\frac{0.0148 \ln (\mathrm{PGA})^{2}+1.2685 \ln (\mathrm{PGA})+0.5515}{0.7103^{2}}\right]
$$

$$
P_{f}\left(L S_{2}\right)=\Phi\left[\frac{0.0148 \ln (\mathrm{PGA})^{2}+1.2685 \ln (\mathrm{PGA})+0.2296}{0.7103^{2}}\right] \text {, }
$$

$$
P_{f}\left(L S_{3}\right)=\Phi\left[\frac{0.0148 \ln (\mathrm{PGA})^{2}+1.2685 \ln (\mathrm{PGA})-0.9999}{0.7103^{2}}\right] \text {, }
$$

$$
P_{f}\left(L S_{4}\right)=\Phi\left[\frac{0.0148 \ln (\mathrm{PGA})^{2}+1.2685 \ln (\mathrm{PGA})-1.0743}{0.7103^{2}}\right] .
$$

The vulnerability curves of different limit states $\left(L S_{i}\right)$ are obtained by plotting PGA and $P_{f}$ in the same rectangular coordinate system. Figure 7 shows the vulnerability curve of each control section of RC pier and RC/ECC pier.

It can be seen from Figure 7 that the exceedance probability of RC pier and high-strength reinforced RC/ECC pier increases with the increase of PGA. The exceedance probability of minor damage and moderate damage of RC pier body is more than $80 \%$, and the exceedance probability of severe damage and complete failure is about $20 \%$. For RC/ ECC piers, the probability of minor damage exceeding is about $75 \%$, the probability of moderate damage exceeding is $61 \%$, and the probability of severe damage and complete failure exceeding is about $10 \%$. Jia et al. [17] carried out a pseudostatic vibration test on the seismic performance of ECC piers in plastic hinge regions. It is concluded that ECC can increase the energy dissipation capacity of pier by $20 \%$ and the displacement ductility coefficient by $15.2 \%$. ECC can obviously improve the failure mode and seismic performance of bridge piers. This is close to the conclusion of seismic vulnerability analysis of piers using ECC in plastic hinge regions in this paper.

In general, the exceedance probability of RC/ECC bridge pier with high-strength bars in each limit state is significantly reduced, which is because ECC and high-strength bars can enhance the ductility and energy dissipation capacity of piers. The difference of exceedance probability of RC/ECC pier with high-strength bars is larger than that of $\mathrm{RC}$ pier under slight damage and medium failure state, while the difference is smaller than that of RC pier under severe failure and complete failure state. That is to say, the use of 
TABLE 3: Control section damage index of RC and RC/ECC pier.

\begin{tabular}{lccccc}
\hline Limit state (damage index) & Minor damage $\left(\phi_{y}^{\prime}\right)$ & Moderate damage $\left(\phi_{y}\right)$ & Severe damage $\left(\phi_{d}\right)$ & Complete failure $\left(\phi_{m}\right)$ \\
\hline \multirow{2}{*}{ RC pier } & Top & 0.001117 & 0.001290 & 0.005000 & 0.005535 \\
& Bottom & 0.001155 & 0.001340 & 0.005084 & 0.005576 \\
\hline \multirow{2}{*}{ RC/ECC pier } & Top & 0.001604 & 0.001867 & 0.005159 & 0.005614 \\
& Bottom & 0.001114 & 0.001537 & 0.005256 & 0.005662 \\
\hline
\end{tabular}

Table 4: Mean and standard deviation of capacity-demand ratio of the pier bottom section.

\begin{tabular}{lccc}
\hline Peak acceleration PGA/g & $\ln ($ PGA) $(\mathrm{g})$ & Mean $\lambda$ & Standard deviation $\sigma$ \\
\hline 0.2 & -1.6094 & -1.4517 & 0.7103 \\
0.4 & -0.9163 & -0.6107 & 0.7103 \\
0.6 & -0.5108 & -0.0926 & 0.7103 \\
0.8 & -0.2231 & 0.2692 & 0.7103 \\
1 & 0 & 0.5515 & 0.7103 \\
\hline
\end{tabular}

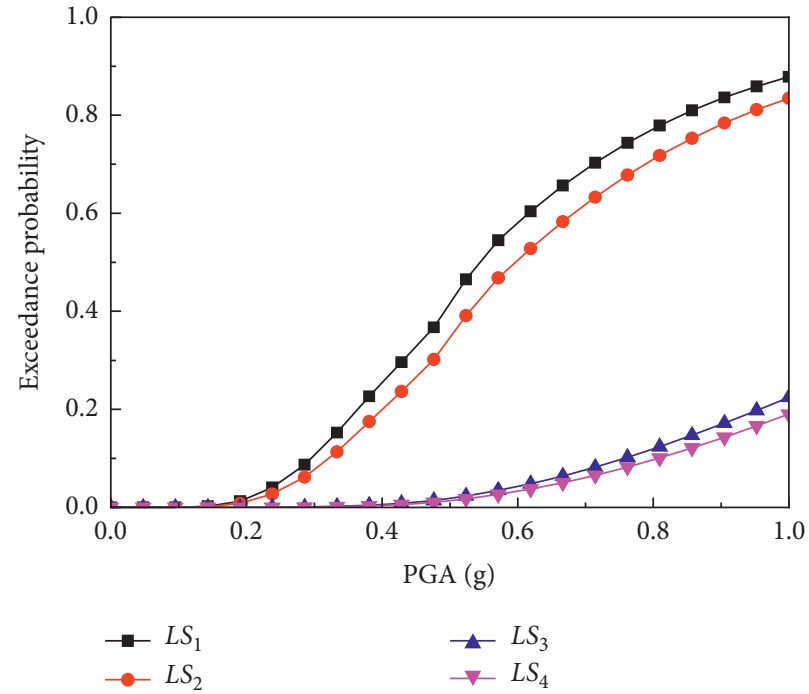

(a)

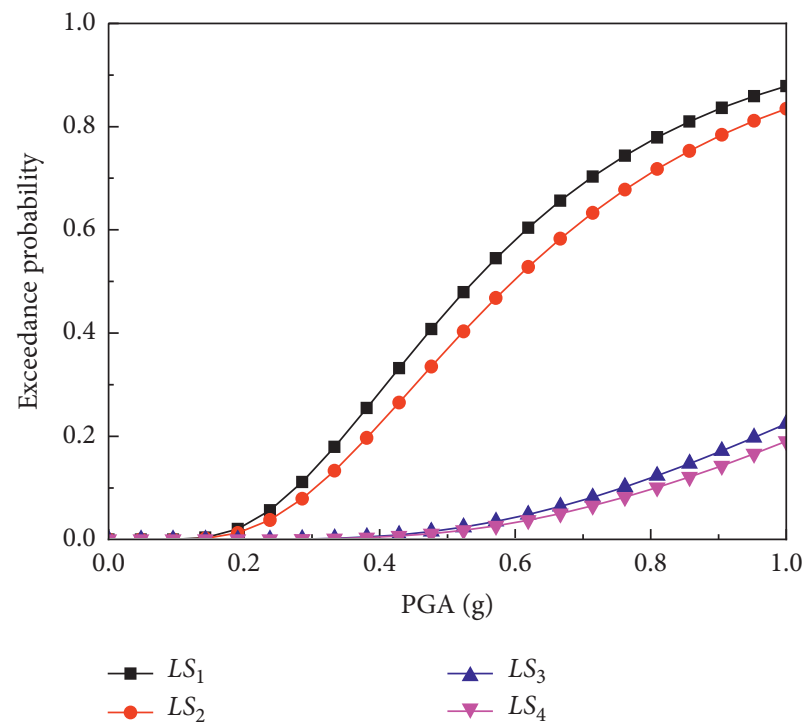

(c)

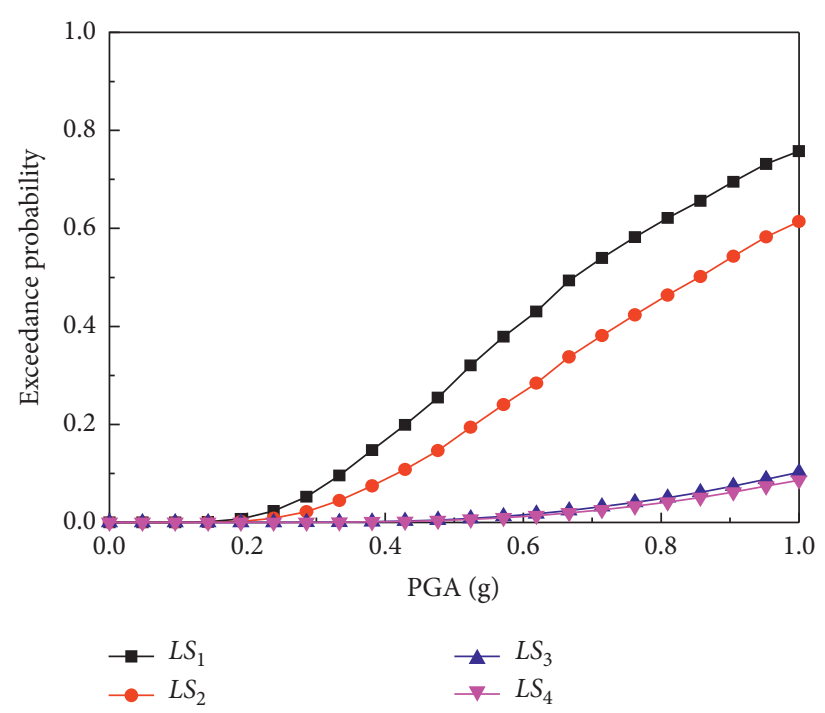

(b)

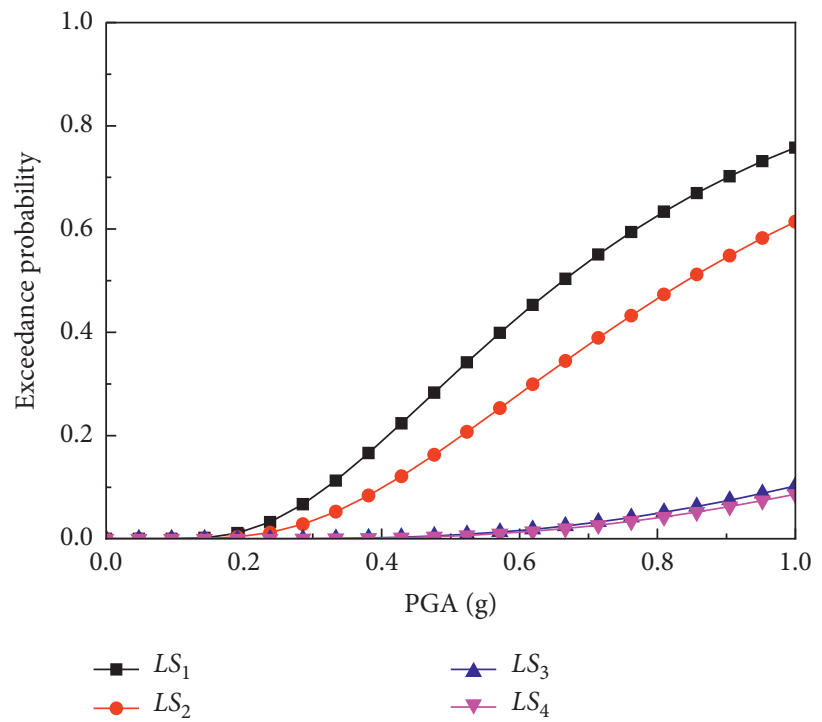

(d)

FIGURE 7: Vulnerability curve of control section: (a) vulnerability curve of RC pier top section, (b) vulnerability curve of RC/ECC pier top section, (c) vulnerability curve of RC pier bottom section, and (d) vulnerability curve of RC/ECC pier bottom section. 
ECC and high-strength bars can reduce the exceedance probability of pier in each limit state and make the difference of exceedance probability more obvious under different limit states. Of course, the results also show that the exceedance probability of failure state has not been improved much. It is consistent with Chen's [21] research conclusion that the seismic performance of piers is improved by increasing the steel bars grade, but the improvement of seismic performance of piers under strong earthquakes is limited. Zheng et al.'s research [22] has come to a similar conclusion.

\section{Summary and Conclusion}

In this paper, the seismic vulnerability of rigid pier with ECC replacing concrete in the plastic hinge regions and longitudinal high-strength bars is analyzed. For the seismic design of ECC in the plastic hinge regions and the engineering application of high-strength bars in the piers, the following conclusions are drawn:

(1) Using ECC to replace concrete in piers can significantly reduce the seismic response of piers under small and medium earthquakes. The maximum curvature of pier is different with different height of concrete replaced by ECC in plastic hinge regions. However, for the two kinds of piers, replaced by ECC at the plastic hinge regions and the whole height, the difference of maximum curvature is very small. Therefore, considering the economy, it is enough to replace the concrete in the plastic hinge regions with ECC to significantly improve the seismic capacity of piers in the design.

(2) In this paper, according to the dynamic time-history analysis of the pier with different volume stirrup ratio and different replacement ratio of highstrength bars in the section, it is found that the economic and reasonable volume stirrup ratio is $0.78 \%$. The seismic performance is the best when high-strength bars replace the longitudinal steel bars in the whole section of the pier.

(3) Under the condition of minor damage and moderate damage, the difference of exceedance probability of high-strength bars RC/ECC pier is bigger than that of RC pier. However, the difference of exceedance probability is smaller than that of RC pier under the condition of severe damage and complete failure. For the pier in the paper, under the condition of slight damage, the exceedance probability of RC pier and RC/ECC pier is $90 \%$ and $75 \%$, respectively. Under the medium failure state, the exceedance probability of RC pier and RC/ECC pier is $80 \%$ and $61 \%$, respectively. Under severe failure and complete failure states, the exceedance probability of RC pier and RC/ ECC pier is $20 \%$ and $10 \%$, respectively. That is to say, ECC material and high-strength steel bar can reduce the exceedance probability of pier in each limit state, making the difference of exceedance probability more obvious in different limit states. It is because
ECC and high-strength bars can enhance the ductility and energy dissipation capacity of piers.

(4) Whether for the top or bottom control section of pier, the seismic performance of high-strength bar $\mathrm{RC} / \mathrm{ECC}$ pier is superior, and the risk of seismic damage is significantly reduced. The decrease of exceeding probability is mainly due to the increase of ductility and energy dissipation capacity of piers with the addition of ECC materials and high-strength steel bars. The appropriate use of high-strength bars in piers is conducive to structural seismic resistance.

\section{Data Availability}

All data generated or analyzed during this study are included within this article. The data used to support the study can be made available upon request to the corresponding author.

\section{Conflicts of Interest}

The authors declare that there are no conflicts of interest.

\section{Acknowledgments}

This work was supported by the Key R\&D and Promotion Projects in Henan Province of China (no. 192102310227).

\section{References}

[1] Ministry of Transport of the People's Republic of China, Detailed Rules for Seismic Design of Highway Bridges, People's Communications Publishing House, Beijing, China, 2008.

[2] V. C. Li and C. K. Y. Leung, "Steady-state and multiple cracking of short random fiber composites," Journal of Engineering Mechanics, vol. 118, no. 11, pp. 2246-2264, 1992.

[3] S. Yao, L. Dai, and L. Ma, "Review on the development of research and engineering properties of engineered cementitious composites," Concrete, vol. 11, pp. 87-91, 2010.

[4] D. Yi, Experimental Study on Theory and Application of ECC Materials, China General Institute of Metallurgical Architecture, Beijing, China, 2008.

[5] G. A. Keoleian, A. Kendall, J. E. Dettling et al., "Life cycle modeling of concrete bridge design: comparison of engineered cementitious composite link slabs and conventional steel expansion joints," Journal of Infrastructure Systems, vol. 11, no. 1, pp. 51-60, 2005.

[6] V. C. Chandler Smith, "Bendable composites: ductile concrete for structures," Structure Magazine, vol. 7, pp. 45-48, 2006.

[7] K. Rokugo, M. Kunieda, and S. C. Lim, "Patching repair with ECC on cracked concrete surface," in Proceedings of the ConMat'05 and Mindess Symposium, pp. 22-24, Vancouver, Canada, August 2005.

[8] Y. Bai, Seismic Vulnerability of Long-Span Concrete Continuous Rigid Frame Bridge with High Piers Based on OpenSees, Southwest Jiaotong University, Chengdu, China, 2016.

[9] S.-Y. Jiang, H.-W. Gong, W.-L. Yao, T. Shuai, and T. Cao, "A survey on mechanical behavior and constitutive model of engineered cementitious composite," Materials Reports, vol. 32, no. 12, pp. 4192-4204, 2018.

[10] A. Neuenhofer and F. C. Filippou, "Geometrically nonlinear flexibility-based frame finite element," Journal of Structural Engineering, vol. 124, no. 6, pp. 704-711, 1998. 
[11] C.-C. Zhang, S.-S. Zheng, and L. Lei, "Flexibility-based fiber beam-column element and its parametric analysis," Industrial Construction, vol. 40, no. 12, pp. 90-94, 2010.

[12] L.-C. Fan and W.-D. Zhuo, Seismic Design of Bridge Ductility, People's Communications Publishing House, Beijing, China, 2001.

[13] L.-Y. Nie, J.-Z. Li, and L.-C. Fan, "Elastic-plastic fiber beamcolumn element and its parametric analysis," Engineering Mechanics, vol. 21, no. 3, pp. 15-20, 2004.

[14] Y. Liang, J. Li, and X.-Y. Lou, "Study on anti-seismic dynamic property of corroded reinforced concrete member," Journal of Vibration Engineering, vol. 29, no. 1, pp. 140-147, 2016.

[15] Y. Liang, J. Li, and X.-Y. Luo, "Performance-based timevarying aseismic design method for durability damaged RC members," Journal of Vibration and Shock, vol. 37, no. 3, pp. 112-118, 2018.

[16] J.-M. Huang, L.-F. Li, and J.-C. Sun, "Research on allowable displacement of high piers based on incremental dynamic analysis," Highway, vol. 2, no. 2, pp. 1-7, 2004

[17] J. Yi, R.-D. Zhao, P. Liao, F.-H. Li, and Y.-L. Zhan, "Experimental investigation on seismic behavior of bridge piers with polypropylene-engineered cementitious composite in plastic hinge regions," China Journal of Highway and Transport, vol. 32, no. 7, pp. 100-110, 2019.

[18] Y. Pan, A. K. Agrawal, M. Ghosn, and S. Alampalli, "Seismic fragility of multispan simply supported steel highway bridges in New York State. II: fragility analysis, fragility curves, and fragility surfaces," Journal of Bridge Engineering, vol. 15, no. 5, pp. 462-472, 2010.

[19] Federal Emergency Management Agency of the United States, Multi-Hazard Loss Estimation Methodology: HAZUS99 User's Manual, FEMA, Washington, DC, USA, 1999.

[20] H. Niu, Time-Varying Seismic Vulnerability Analysis of RC Rigid Frame Piers in Offshore Environment, Zhengzhou University, Zhengzhou, China, 2018.

[21] Y.-W. Chen, "Study on the effect of material parameters on the seismic fragility performance of high-speed railway continuous beam bridge," Railway Standard Design, vol. 61, no. 7, pp. 88-92, 2017.

[22] S.-S. Zheng, K.-Y. Dai, and C.-W. Han, "Steel bent frame structure vulnerability analysis based on steel performance degradation model," Journal of Vibration and Shock, vol. 34, no. 17, pp. 18-24, 2015. 